\title{
Intestinal epithelial barrier: The target for pathogenic Escherichia coli
}

\author{
Barbara Pawłowska ${ }^{1, A, D}$, Beata M. Sobieszczańska ${ }^{1, A, D}$ \\ ${ }^{1}$ Department of Microbiology, Wroclaw Medical University, Poland \\ A - research concept and design; B - collection and/or assembly of data; $\mathrm{C}$ - data analysis and interpretation; \\ $\mathrm{D}$ - writing the article; $\mathrm{E}$ - critical revision of the article; $\mathrm{F}$ - final approval of the article
}

\section{Beata M. Sobieszczańska \\ Funding sources \\ None declared \\ Conflict of interest \\ None declared}

Address for correspondence

E-mail: beata.sobieszzzanska@umed.wroc.pl

Received April 6, 2016

Reviewed June 23, 2016

Accepted August 29, 2016

\begin{abstract}
Diarrheagenic Escherichia coli strains are included in 9 pathotypes (pathovars) that present different virulence factors responsible for the patomechanism of infections they cause. As all other intestinal pathogens, E. coli exerts a significant effect on intestinal epithelium. To initiate the infection, these microorganisms have evolved countless strategies to subvert the epithelial barrier and efficiently colonize the intestinal epithelium. The barrier function of the intestinal epithelium is achieved by the presence of a tight junction protein network surrounding individual cells around their circumference that links neighboring cells and seals the intracellular space. Pathogenic E. coli strains may impair intestinal epithelial barrier in 3 different pathways: (i) through a direct effect of their virulence factors on tight junctions proteins, (ii) by disrupting host cell actin cytoskeleton that indirectly damages epithelial barrier, and (iii) via stimulation of the secretion of proinflammatory cytokines that directly disrupt epithelial tight junctions or trigger neutrophils migration through intestinal epithelium, thus disrupting the intestinal barrier. Most pathogenic E. coli generates all these 3 pathways concomitantly upon interaction with intestinal epithelium.
\end{abstract}

Key words: Escherichia coli, intestinal barrier, tight junctions

DOI

$10.17219 /$ acem/64883

\section{Copyright}

Copyright by Author(s)

This is an article distributed under the terms of the

Creative Commons Attribution Non-Commercial License

(http://creativecommons.org/licenses/by-nc-nd/4.0/) 


\section{The intestinal epithelial barrier}

The epithelium lining the gastrointestinal tract makes up the largest interface between the external and internal milieu in human organism. This single columnar cell layer provides a selectively permeable barrier that allows nutrients, water, and electrolytes to be absorbed, but simultaneously prevents the passage of luminal antigens like toxins and microbial flora to the lamina propria and to the bloodstream. ${ }^{1,2}$ Furthermore, armed with the secretory immunoglobulin A (sIgA), Paneth cells that produce antibacterial peptides as well as immune cells, i.e., dendritic cells, mast cells, macrophages, and B and $\mathrm{T}$ cells localized beneath the epithelial cells in lymphoid follicles, the intestinal epithelium, serve as a first line of the host's innate immune defense. ${ }^{3,4}$

The barrier function of the intestinal epithelium is achieved by the presence of a protein network surrounding individual cells around their circumference that links adjacent cells and seals the intracellular space. ${ }^{1}$ The protein connections between adjacent cells are composed of 2 complexes including the apical junction complex (AJC) that forms 2 separate zones, i.e., tight junction (TJ) and the adherens junction (AJ), and desmosomes localized at the basolateral membranes, which support epithelial stability (Fig. 1). Both TJ and AJ form 2 extracellular loops in the paracellular space that interlock the same structures of the neighboring cell. At the other end, $\mathrm{TJ}$ and $\mathrm{AJ}$ are anchored directly to the F-actin cytoskeleton (Fig 1). The main role of AJC is to polarize epithelial cells by separating the apical membrane above the AJC that faces the intestinal lumen and the basolateral membrane below AJC which is in contact with the lamina propria. ${ }^{1,5}$
$\mathrm{TJ}$ is the most apical complex connecting and sealing the intercellular space between adjacent cells. This highly specialized network of proteins is composed of key transmembrane barrier proteins, such as occludin, claudins and junctional adhesion molecules (JAMs) linked to the peripheral membrane (scaffolding) proteins (e.g., zonula occludens ( $\mathrm{ZO})$, afadin) that are in turn connected with actin and microtubules by linkers, e.g., cingulin and non-muscle myosin. Myosin and actin, collectively referred to as actomyosin, form a ring encircling the cell at the level of AJC (Fig. 2). ${ }^{6}$

Claudins are the family of at least 24 important proteins essential for barrier function that form paracellular ionselective channels across TJs. They have 4 transmembrane domains that form 2 loops in the extracellular space specifying their ion-selective channel functions. These extracellular loops of claudins of neighboring cells appearing opposite to each other are linked together forming $\mathrm{TJ}$ strands. The largest $1^{\text {st }}$ loop is considered to be critical for determining the paracellular tightness and selective ion permeability, whereas the $2^{\text {nd }}$ one has a holding function between opposing cell membranes, although it may also narrow the paracellular cleft. ${ }^{7}$ The cytoplasmic C-terminus of claudins is linked to the peripheral membrane adaptor $\mathrm{ZO}$ proteins of the cytoplasmic plaque (i.e., ZO-1, -2 and -3 proteins). ZO proteins link TJs to the actin and microtubule cytoskeleton (Fig. 2). Different claudin isoforms are expressed simultaneously in all epithelial tissues, and exhibit distinct expression patterns specific to the tissue and cell types. . $^{6,9} 9$

Occludin also forms 2 loops in the extracellular space with $\mathrm{N}$ - and $\mathrm{C}$-terminal domains anchored in the host cell cytosol that are important in the sealing of the TJ. Phosphorylation of long C-terminal domain via cellular

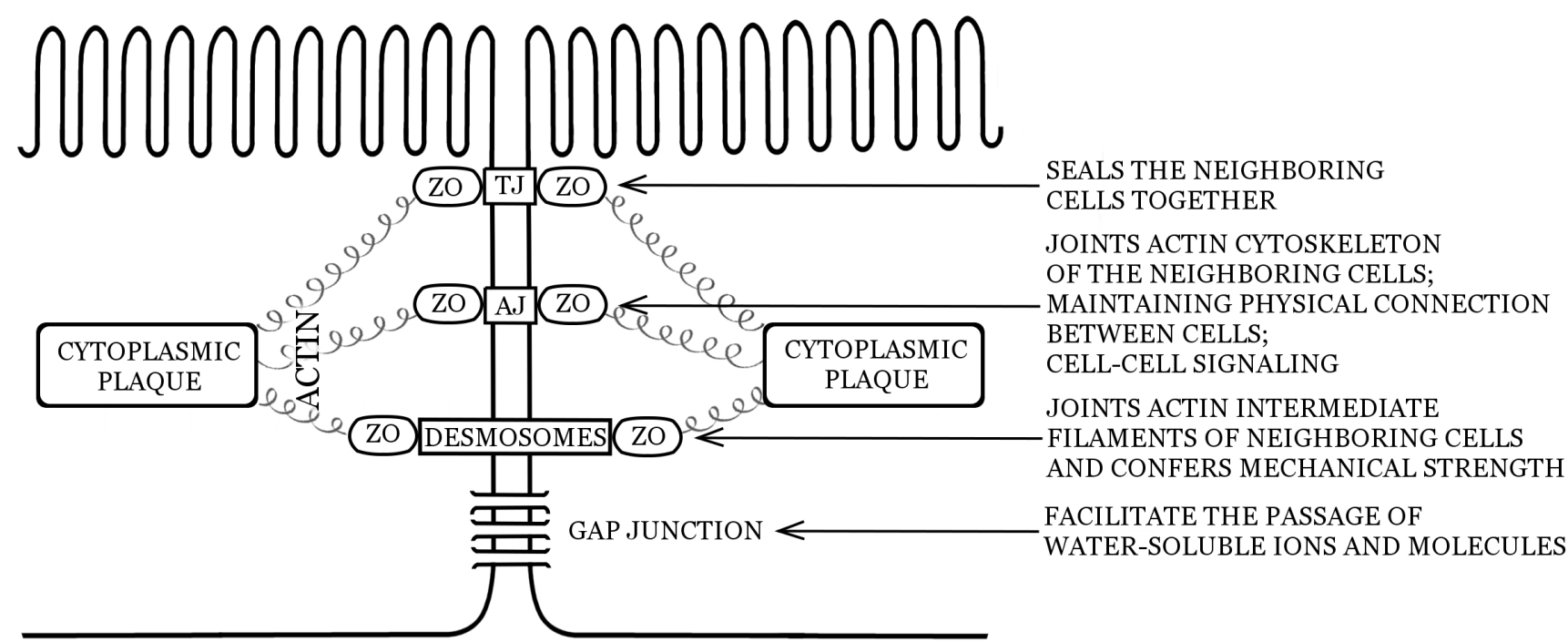

Fig. 1. Main types of cell junctions 
kinases or phosphatases regulates occludin's localization in the TJ and influences occludin-ZO-1 interactions, and thus barrier function maintenance. Moreover, occludin interacts with claudins and JAM-A. ${ }^{10}$ By multiple interaction with many signaling molecules, occludin play a role in signal transduction. ${ }^{8}$

JAMs transmembrane glycoproteins belong to an immunoglobulin (Ig) superfamily and play a role in regulating epithelial cell polarity as well as in leukocytesepithelial or endothelial cells interactions. ${ }^{8,11}$ There are 3 JAMs isoforms, i.e., JAM-A, -B, and -C, that can form homophilic and heterophilic interactions at the adhesion points via PDZ-binding domain at their cytoplasmic portion. PDZ domain is a common structural domain for binding to a short motif at C-terminal domain of other protein. JAM-A together with E-cadherin and ZO-1 localizes in a spot-like adherens junctions at early cell-cell fusion point. As a result, contacting cells start to polarize via formation of TJs and AJs. ${ }^{11,12}$

Cytosolic TJ plaque proteins comprise 2 main categories: (i) peripheral proteins (e.g., $\mathrm{ZO}-1,-2,-3$, cingulin) that organize transmembrane proteins and link them to other cytoplasmic proteins as well as to actin cytoskeleton; and (ii) signaling proteins involved in TJs assembly. ${ }^{10}$

AJ through its interactions with F-actin network is responsible for the maintenance of the physical connection between neighboring cells and is thus important in cellcell signaling. AJ consists of transmembrane proteins (cadherins) and adaptor proteins. Cadherins are calciumdependent cell adhesion molecules that are divided into classical cadherins, e.g., E-cadherin, N-cadherin, P-cadherin, desmosomal cadherins, protocadherins, and nonconventional cadherins. ${ }^{13}$ Highly conserved cytoplasmic domains of classical cadherins interact with cytoplasmic proteins, i.e., $\beta$-catenin that in turn bind to $\alpha$-catenin, which is linked to actin-binding proteins. ${ }^{14}$ Thus, all these cytoplasmic components of AJ play an important role in the strength and stability of cell-cell contact and epithelial polarity (Fig. 3). ${ }^{13,15}$

Desmosomes are intercellular junctions that make focal connections between intermediate keratin filaments of neighboring cells. Due to their characteristic structure, desmosomes confer tensile strength and resilience to cells. Morphologically, desmosomes are highly organized, and they consist of a central core region between opposing cells and 2 identical cytoplasmic plaques that are associated with the cytoskeleton network. As previously clarified, all desmosomes are composed of desmoplakin, plakoglobin, at least 1 isoform each of plakophilin and the desmosomal cadherins: desmocollin and desmoglein (Fig. 4). ${ }^{16}$ Additionally, various accessory proteins are involved in desmosomal adhesion. ${ }^{17}$

Under physiological conditions, FJ, TJ, AJ and desmosomes link adjacent cells and cooperate together to maintain the intestinal epithelial barrier function. However, direct contact of intestinal epithelial cells and their junc- tional complexes with luminal content exposes the epithelium to a number of pathogenic microorganisms which gain access to the intestines with food and water.
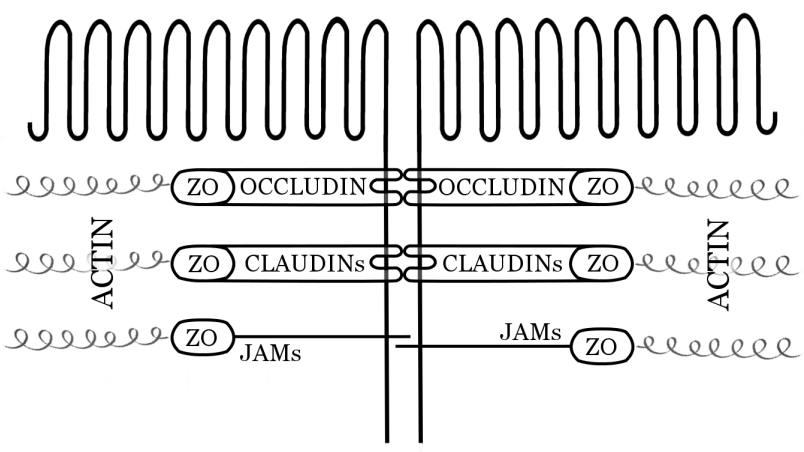

Fig. 2. The simplified architecture of tight junction (TJ)

ZO - zonula occludens; JAMs - junctional adhesion molecules.

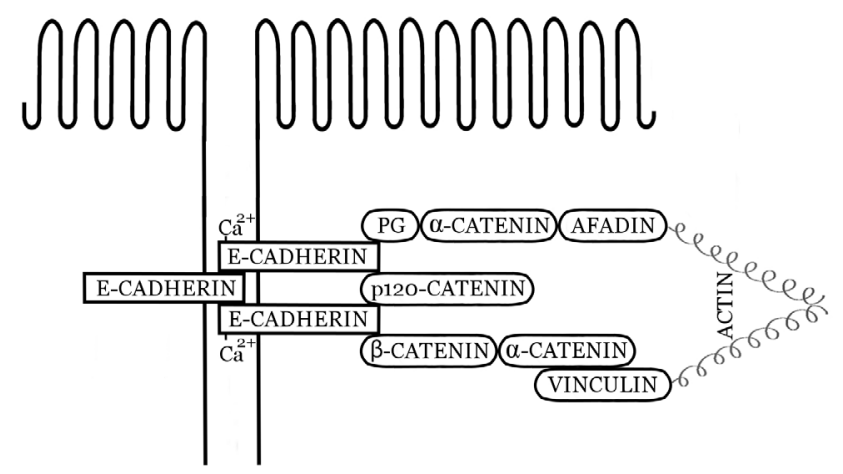

Fig. 3. The simplified architecture of adhernes junction (AJ)

PG - plakoglobin; AJs are located below TJs in the apical part of the basolateral membrane and comprise $\mathrm{Ca}^{2+}$ - dependent adhesion molecules cadherins. Cadherins attach to catenins and then the complex is linked to actin filaments via vinculin. p120 catenin interacts with juxtamembrane domains of cadherins participating in the cell-cell adhesion.

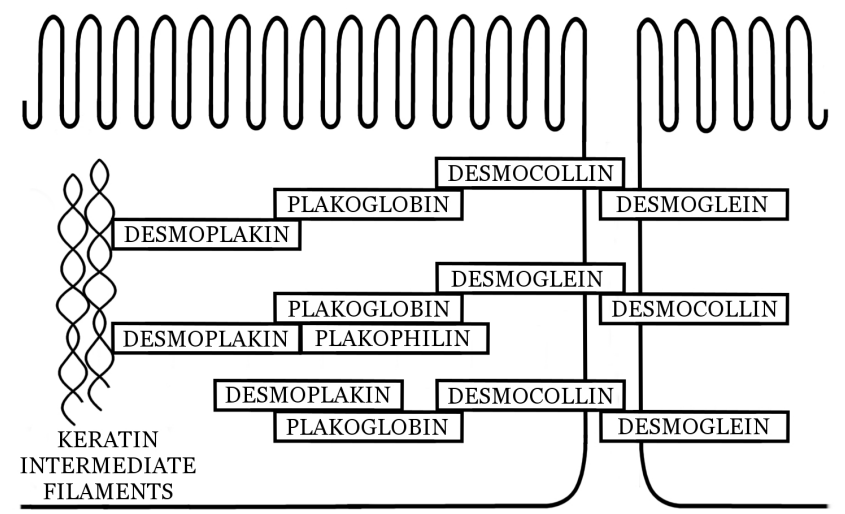

Fig. 4. A simplified schematic diagram of desmosomes 


\section{How pathogenic Escherichia coli induces intestinal epithelial barrier disturbances}

Intestinal pathogens have evolved countless strategies to interfere with junctional complexes and to cross the epithelial layer. Over the recent years, the association between the dysfunction of the intestinal barrier and the development of Crohn's disease, ulcerative colitis, and microbial infections has been discovered. ${ }^{18}$ One of the best-known pathogens responsible for gastrointestinal tract infections or combined with idiopathic intestinal diseases, e.g., inflammatory bowel disease or bowel cancers, are pathogenic Escherichia coli (E. coli) strains.

Diarrheagenic E. coli strains belong to 9 pathotypes, each one of which has a characteristic set of virulence factors responsible for different patomechanism of infections they cause. These pathotypes include enteropathogenic E. coli (EPEC), enterohemorrhagic E. coli (EHEC), shiga toxin-producing $E$. coli (STEC), enterotoxigenic E. coli (ETEC), enteroinvasive E. coli (EIEC), enteroaggregative E. coli (EAEC), diffusely-adhering $E$. coli (DAEC), cell-detaching $E$. coli (CDEC), and adherent-invasive E. coli (AIEC). All these pathotypes can impair intestinal barrier integrity upon infection.

Intestinal epithelial barrier disruption is mediated by pathogenic E. coli in many different ways, e.g., upon adhesion, via toxins or effector proteins delivered directly to the host cells, or is induced by cytokines stimulated upon infection with these pathogens. ${ }^{19}$

\section{Enteropathogenic E. coli and enterohemorrhagic $E$. coli - Host cell actin cytoskeleton modulators}

Enteropathogenic E. coli is a common cause of gastroenteritis in infants, whereas enterohemorrhagic E. coli causes bloody diarrhea in humans that may progress to hemolytic urinary syndrome (HUS). Both EPEC and EHEC, as well as Citrobacter rodentium and rabbit EPEC (REPEC), are pathogenic for animals, and they all share a common patomechanism involving the production of characteristic histopathological lesions A/E (attaching and effacing) in the host's intestinal epithelium and are, therefore, collectively named A/E pathogens. The adherence and tight attachment of A/E pathogens to enterocytes induces a cascade of morphological and structural alterations in the epithelial cells involving localized destruction and collapse (effacement) of brush border microvilli. The development of the A/E lesions is also combined with the accumulation of filamentous actin that together with $\alpha$-actinin, ezrin, talin, and myosin form a pedestal-like structures beneath the adhering bacteria. ${ }^{19}$
Decreased transepithelial electrical resistance (TEER) of polarized epithelial cells, indicating decreased epithelial permeability that accompanies infections caused by $A / E$ pathogens initiated research on the influence of $\mathrm{A} / \mathrm{E}$ pathogens on TJs. These studies demonstrated that $\mathrm{A} / \mathrm{E}$ pathogens subvert the host's intestinal permeability barrier in many different pathways, but intimate contact of the pathogen with the host cell is crucial in the process.

The process is initiated immediately after the attachment of $\mathrm{A} / \mathrm{E}$ pathogen to the enterocyte by the injection of effector proteins through syringe-like apparatus, i.e., a type III secretion system (TTSS) directly to the host cell cytozol. Translocated intimin receptor (Tir) delivered through TTSS to the apical cytoplasmic membrane of enterocytes is one of the most important effectors modulating actin cytoskeleton of the host cells. Interaction of Tir inserted to the host cell cytoplasmic membrane, by means of the adhesion molecule intimin in the outer membrane of A/E pathogens, triggers Tir phosphorylation by host tyrosine kinases, e.g., Src family kinase c-Fyn, followed by the recruitment of the adaptor signaling protein Nck, which in turn recruits neuronal Wiskott-Aldrich Syndrome protein (N-WASP) and the actin-relating protein Arp2/3 complex. ${ }^{19,20} \mathrm{~N}$-WASP is a member of the WASP family of proteins that signal to the host cell cytoskeleton through the Arp2/3 complex, nucleating new actin filaments and cross linking existing filaments into actin networks. Activation of N-WASP-Arp2/3 complex mediates actin pedestals formation, i.e., histopathological lesions A/E beneath the adhering bacterium. Moreover, it has been shown that Tir of EPEC can also bind to the host cell multidomain protein IQGAP1, a well-known regulator of the cy-

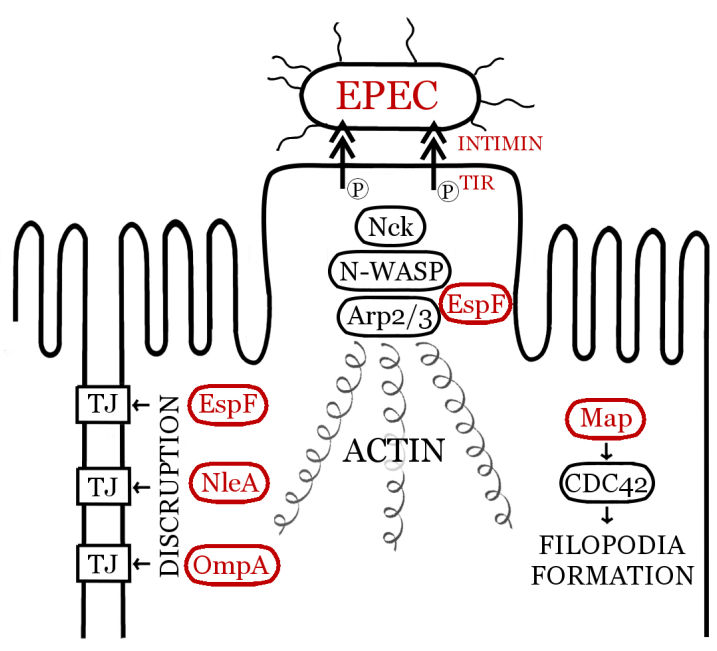

Fig. 5. The impact of EPEC on epithelial barrier

Adherence of enteropathogenic E. coli (EPEC) to the small bowel enterocytes induces the development of characteristic actin-rich pedestals beneath adherent bacteria which is associated with the collapse of microvilli. 
toskeleton, involved in Rho family GTPases Rac1/Cdc42 and $\mathrm{Ca}^{2+} /$ calmodulin signaling, and actin polymerization. ${ }^{19-21}$ On the other hand, Brown et al. also demonstrated that although cells lacking protein IQGAP1 have significantly attenuated actin polymerization in response to EPEC attachment, the knockout of IQGAP1 did not abrogate pedestal formation. ${ }^{20}$ Peralta-Ramirez et al. in their study of REPEC strain demonstrated that EspF protein secreted by A/E pathogens via TTSS to a host cell is involved in the regulation of actin polymerization by binding to a complex of proteins, e.g., N-WASP and Arp2/3, but also to ZO-1, ZO-2 proteins at the TJs. Furthermore, EspF caused claudin, occludin, ZO-1 and $\mathrm{ZO}-2$ proteins redistribution and their recruitment into the actin pedestals. ${ }^{22}$ These authors suggested that EspF may cause local actin depolymerization and thus EspFinduced TJ disruption.

The disturbances in the host cell actin cytoskeleton induce the contraction of actin filaments attached to the TJs pulling the junction opened. ${ }^{23}$ Similar results were demonstrated by Muza-Moons et al., who confirmed the crucial role of EspF protein in TJs disruption. ${ }^{24}$ In their study, T84 epithelial cells infected with EPEC progressively lost interaction of occludin and claudin-1 with the cytosolic plaque protein ZO-1. Mutation of the gene encoding EspF prevented the disruption of TJs. Alto et al. have shown that EspF localizes to membrane trafficking organelles and nucleates a multiprotein signaling complex consisting of eukaryotic sorting nexin 9 (SNX9) and N-WASP involved in multiple host cellular regulatory pathways, e.g., membrane trafficking, actin-remodeling, and the overall epithelial homeostasis. ${ }^{25}$ Their study indicated that EspF is necessary, although not sufficient, to regulate $\mathrm{TJ}$ architecture during EPEC infection. On the other hand, Malladi et al. have shown that other virulence factors of EPEC may contribute to intestinal barrier disruption. ${ }^{26}$ These authors demonstrated that outer membrane proteins (OMPs) of EPEC activate protein kinase $\mathrm{C}(\mathrm{PKC})$, which is associated with phosphorylation of cadherins and leads to the dissociation of the cadherin/ $\beta$-catenin complex from AJ. Additionally, 2 other proteins of EPEC, i.e., mitochondrial associated protein (Map) and NleA (EspI) cause barrier disruption by perturbing TJs. It has been shown that Map is a guanidine-nucleotide exchanging factor (GEF) for CDC42, a small GTPase of the Rho family that regulates actin dynamics through binding to N-WASP. NleA disrupts COPII, a coat protein complex involved in trafficking membrane proteins from endoplasmic reticulum, thus blocking the delivery of new TJs proteins (Fig. 5). ${ }^{27}$

All these results suggest that A/E pathogens via TTSS secreted effector proteins but also other virulence factors may utilize many pathways impairing barrier integrity to promote effective colonization of intestinal epithelium. In turn, disturbances in epithelial permeability contribute to the development of secretory diarrhea and thus provide the exit and the way of spreading for $\mathrm{A} / \mathrm{E}$ pathogens to new hosts.

\section{Enteroaggregative E. coli}

Enteroaggregative E. coli strains differ from other pathogenic $E$. coli characteristic aggregative adherence pattern to epithelial cells (Fig. 6).

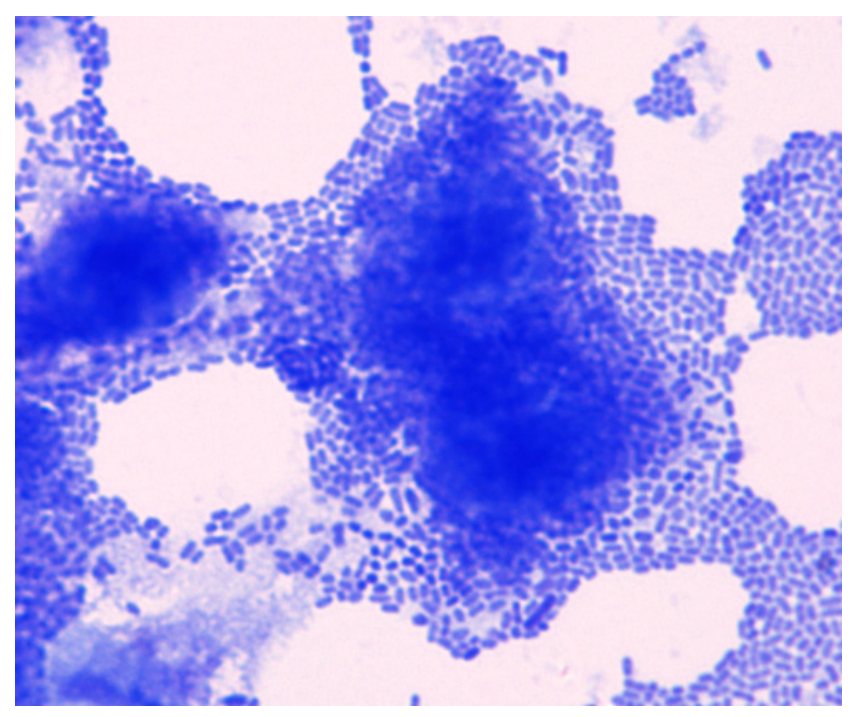

Fig. 6. Aggregative adherence of EAEC to HEp-2 cells

In the picture there are visible epithelial cells totally covered with adherent bacteria. Giemsa stain, magnification $\times 100$

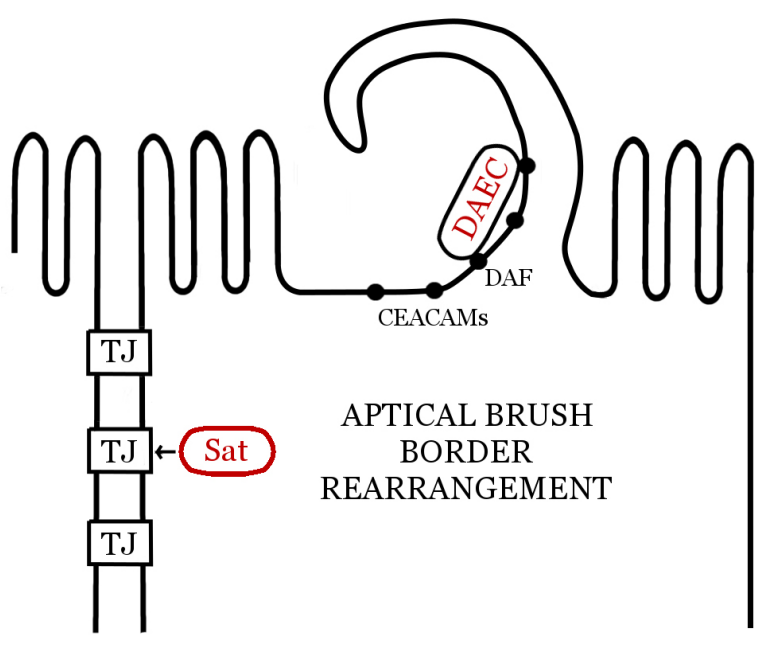

Fig. 7. Epithelial barrier disruption by DAEC

CEACAMs - carcinoembryonic antigens; DAF - decay accelerating factor; both CEACAMs and DAF serve as anchoring molecules on the surface of intestinal epithelium for binding diffusely adhering $E$. coli (DAEC). Effector proteins of DAEC induce specific apical brush border rearrangement leading to the formation of a long finger-like edgings that wrap the adherent bacteria. 
These pathogenic E. coli strains are responsible for acute and chronic diarrhea among children and adults all over the world. ${ }^{28}$ Strauman et al. demonstrated on in vivo intestinal cell model that EAEC O42 and JM221 reference strains induced decrease in epithelial TEER via aberrant localization of the tight junction proteins occludin, ZO-1, and claudin-1. ${ }^{28}$ They also found that the effect was induced by aggregative adherence fimbriae I and II (AAF/I and AAF/II) specific to EAEC pathotype as afimbrial mutant strains did not impair the TJ integrity. Involvement of claudin- 1 is a common theme in the enteric infections, as several intestinal pathogens, including EHEC and Salmonella enterica serovar Typhimurium, cause a redistribution of claudin-1 in addition to disrupting paracellular permeability. ${ }^{28}$ Moreover, EAEC can disrupt intestinal epithelial integrity via the action of Pet, a serine protease autotransporter enterotoxin, that cleaves the actin-binding protein $\alpha$-fodrin within the cytoskeleton, leading to cell rounding and detachment from the substratum. ${ }^{29}$ Pet enterotoxin is a member of serine protease autotransporter proteins of Enterobacteriaceae (SPATE) that have multiple effects on host cell cytoskeleton and cell-cell connections.

\section{Diffusely adhering $E$. coli}

Diffusely adhering E. coli strains presenting Afa/Dr fimbrial and afimbrial adhesins produce Sat toxin, another member of serine protease autotransporters, which causes an increase in paracellular permeability and the rearrangement of the tight junction proteins of $\mathrm{ZO}-1$, $\mathrm{ZO}-2$, ZO-3, occludin, and claudin-1 that are linked to the cytoskeleton and play a pivotal role in the TJ architecture. ${ }^{19,30,31}$ Another serine protease of SPATE family, i.e., EspC secreted by EPEC through TTSS, contributes indirectly to the alteration of epithelial barrier via the destruction of the actin cytoskeleton. Similarly to Pet enterotoxin of EAEC, EspC cleaves $\alpha$-fodrin (spectrin) that links actin filaments with the host cell plasma membrane, and thus maintains the stability of cellular cytoskeleton and its mechanical properties. Loss of $\alpha$-fodrin disrupts the structural link between actin cytoskeleton and plasma membrane, leading to the collapse of cytoskeleton and TJs disruption with resulting influx of luminal antigens into the submucosa (Fig. 7). ${ }^{19}$

Irrespective of the Sat, Peiffer et al. demonstrated that the reference Afa/Dr DEAC C1845 strain causes selective lesions in the intestinal epithelial barrier producing increased paracellular permeability to mannitol but no change in the paracellular passage of nonionic molecules which have higher molecular masses. ${ }^{31}$ This phenomenon was accompanied by a dramatic alteration in the distribution of TJ-associated occludin and ZO-1 protein, and was independent of apical cytoskeleton rearrangements produced by the strain. However, the bacterial factor contributing to the alteration of TJ proteins is still unknown. ${ }^{31}$ Apart from the impact of DEAC on host cell junctions, the adherence of these strains to the intestinal epithelial cells produces prominent brush border

Table 1. The influence of pathogenic E. coli on the intestinal epithelial barrier

\begin{tabular}{|c|c|c|}
\hline E. coli pathotype & Virulence factor & Effect on epithelial barrier \\
\hline AIEC & type 1 pili & $\begin{array}{l}\text { up-regulation of CEACAM6 associated with abnormal expression of claudin-2, } \\
\text { ZO-1, occludin, and E-cadherin; disorganization of actin cytoskeleton }\end{array}$ \\
\hline \multirow{5}{*}{ EPEC/EHEC } & EspF effector protein & redistribution of claudin, occludin, ZO-1, and ZO-2 leading to TJ disassembly \\
\hline & Map protein & reduced host cell proteins trafficking; TJs disassembly \\
\hline & NleA (Espl) protein & $\begin{array}{l}\text { inhibition of host cell protein trafficking and blocking the delivery of a new } \\
\text { TJs proteins }\end{array}$ \\
\hline & EspC protein & $\begin{array}{c}\text { cleavage of a-fodrin linking actin filaments with cell membrane, leading } \\
\text { to the cytoskeleton collapse and TJs disruption }\end{array}$ \\
\hline & outer membrane protein A (OmpA) & $\begin{array}{l}\text { activation of protein kinase } C(\mathrm{PKC}) \text { and dissociation of cadherin/ } \beta \text {-catenin } \\
\text { complex from AJ }\end{array}$ \\
\hline ETEC & STb enterotoxin & redistribution of ZO-1, claudin-1, and occludin proteins \\
\hline \multirow{3}{*}{ DAEC } & Sat serine protease & $\begin{array}{l}\text { rearrangement of } \mathrm{ZO}-1, \mathrm{ZO}-2, \mathrm{ZO}-3 \text {, and claudin and disrupting TJs } \\
\text { architecture }\end{array}$ \\
\hline & unknown factor & $\begin{array}{l}\text { altered distribution of occludin } \\
\text { and ZO-1 protein }\end{array}$ \\
\hline & Afa/Dr fimbriae & $\begin{array}{l}\text { rearrangement of apical cytoskeleton proteins: F-actin, villin, a-actin, ezrin, } \\
\text { tropomyosin }\end{array}$ \\
\hline \multirow{2}{*}{ EAEC } & aggregative adherence fimbriae (type AAFI - III) & $\begin{array}{l}\text { decreased TEER, aberrant localization } \\
\text { of occludin, ZO-1 and claudin-1 }\end{array}$ \\
\hline & Pet serine protease & $\begin{array}{l}\text { cleaves the actin-binding protein } \\
\text { a-fodrin }\end{array}$ \\
\hline EIEC & Ipa and IcsA effector proteins & recruitment of actin-nucleating complex Arp2/3 and N-WASP \\
\hline
\end{tabular}


injury and rearrangement in apical cytoskeleton proteins, i.e., F-actin, villin, $\alpha$-actinin, ezrin, and tropomyosin, thus playing an important role in the organization and maintenance of the brush border integrity. ${ }^{19}$

\section{Enteroinvasive E. coli}

Rearrangement of the host cell action cytoskeleton, along with the disruption of intestinal epithelial barrier integrity, also accompanies infections caused by Enteroinvasive $E$. coli. The pathotype causes an invasive colitis that occasionally presents as dysentery. Through the TTSS, these pathogens secrete multiple effector proteins, i.e., Ipa A, IpaB, IpaC, and IpaD, that are responsible for the EIEC entering into colonocytes, the lysis of the endocytic vacuole and phagosome escaping into host cell cytoplasm, and then cytoskeleton rearrangements necessary for intracellular spread of these organisms. ${ }^{32}$ Many effector proteins of EIEC interact with small Rho GTPases, i.e., Rac1 and Cdc42, which recruit the actin-nucleating complex Arp2/3. In turn, the IcsA, an outer membrane protein of EIEC delivered via TTSS into host cell membrane, interacts with N-WASP at 1 bacterial pole, allowing for EIEC to move through the epithelial cell cytoplasm and spread to the adjacent cells. ${ }^{19}$

\section{Enterotoxigenic E. coli}

Enterotoxigenic E. coli, endemic in most developing countries, is an important cause of watery diarrhea among children and travelers. ETEC characterizes the production of heat-stable (ST) enterotoxins a (STa) and b (STb), and heat-labile (LT) enterotoxins I (LTI) and II (LTII) that disrupt intestinal epithelial cells secretory activity, resulting in diarrhea. ${ }^{27}$ Nassour et al. have demonstrated that STb enterotoxin induces intestinal epithelial barrier dysfunction through changes in the tight junction protein claudin-1. ${ }^{33}$ Mukiza et al. have demonstrated that STb internalized by T84 epithelial cells caused a significant reduction of TEER and an increase in paracellular permeability, which was associated with the alteration of F-actin stress fibers. ${ }^{34}$ Changes in F-actin dissolution and fragmentation were related to the redistribution and/or fragmentation of ZO-1, claudin-1, and occludin proteins.

\section{Crohn's associated adherent - Adherent-invasive $E$. coli (AIEC)}

The pathogenesis of Crohn's disease, although still unclear, seems to be multifactorial and related to genetic predisposition and to a dysregulated immune response to altered host intestinal microflora. Increased intestinal permeability observed in patients with Crohn's dis- ease promotes the exposition of intestinal epithelium to luminal content and triggers chronic inflammatory response to intestinal microflora. Adherent-invasive $E$. coli is the pathotype, comprising $E$. coli strains, which characterizes the ability to adhere to and invade epithelial cells and capability to survive and multiply within macrophages. ${ }^{35}$ Although there are several reports presenting evidence that AIEC may be the pathogen triggering Crohn's disease, presently, they are considered to be a pathobiont which intensifies the preexisting inflammation rather than induce this form of inflammatory bowel disease. Nevertheless, it has been shown that AIEC binds via type 1 pili to carcinoembryonic antigen CEACAM6 overexpressed on the epithelial cells of patients with Crohn's disease, enhancing further the expression of CEACAM6. The expression of CEACAM6 is additionally increased by the proinflammatory cytokines, i.e., tumor necrosis factor alpha (TNF $\alpha)$ and interferon gamma (IFNy) produced by macrophages infected with AIEC and lymphocytes. ${ }^{35}$ Denizot et al. demonstrated on a CEABAC10 transgenic mouse model expressing human CEACAMs that barrier disruption precedes a relapse of Crohn's disease in asymptomatic patients. ${ }^{36}$ These authors suggested that up-regulated CEACAMs expression on epithelial cells interfere with other adhesion molecules located between lateral membranes and may disrupt epithelial cells architecture, leading to the development of colitis. Moreover, they also showed that the infection of the CEABAC10 mice led to a 3-fold increase in intestinal permeability and to the disruption of mucosal integrity via interaction of type 1 pili of AIEC with CEACAM6. Interestingly, the process was associated with abnormal expression of claudin-2, disorganization of actin cytoskeleton, and mislocalization of ZO-1 and E-cadherin. ${ }^{36,37}$ Similar results were presented by Wine et al., who demonstrated that an infection of polarized T84 cells with AIEC caused a reduction in TEER and increased dextran flux, both accompanied by the redistribution of the TJ adaptor protein ZO-1. ${ }^{38}$ Moreover, it has been demonstrated that AIEC induces increased expression of claudin-2 and decreased expression of occludin. ${ }^{39}$ Although the direct impact of AIEC infection on polarized epithelial cells induces alterations in epithelial permeability, it is noteworthy that AIEC may induce intestinal barrier disruption indirectly by eliciting strong immune response and release proinflammatory cytokines, a well-known TJs proteins modulator. Infection of intestinal epithelium with AIEC results in the secretion of an array of cytokines, i.e., interleukin-1 $\beta$ (IL-1 $\beta$ ), IL-6, TNF $\alpha$, that may impair intestinal barrier integrity. Similarly to AIEC, other E. coli pathotypes trigger transepithelial migration of dendritic cells and neutrophils as well as an expression and upregulation of monocyte chemotactic protein 1 (MCP-1), macrophage inflammatory protein $3(\mathrm{MIP} 3 \alpha)$, and a number of chemokines, i.e., IL-8, GRO $\alpha$, apart from mentioned above. ${ }^{40}$ 


\section{Summary}

Although the direct impact of pathogenic E. coli on TJs proteins has been demonstrated in several manuscripts, it seems that the main target for these pathogens is the F-actin cytoskeleton of intestinal epithelial cells. Enterocytes presenting a number of glycoproteins provide the adherence sites for pathogenic $E$. coli, thus protecting them from removal via peristalsis. As successful pathogens, pathogenic E. coli must provide themselves a niche to replicate sufficiently. EIEC and AIEC pathotypes can invade and replicate within enterocytes and intestinal macrophages, whereas other remain on the surface of enterocytes which is associated with the necessity to interact with the host immune response. Direct contact of adhering E. coli with the host cells surface triggers the activation of the immune response that can be utilized by some pathogens. Neutrophils migration on the surface of intestinal epithelium upon infection with EIEC is associated with the opening of TJs, facilitating the access of EIEC to the basolateral surfaces and their invasion into enterocytes. A similar strategy can be used by AIEC to reach macrophages in the lamina propria. EAEC pathotype has the unusual ability to form a specific biofilm on the surface of intestinal epithelium that makes these pathogens difficult to remove via peristalsis, but also protecting them from hostile intestinal environment as well as immune system cells. DAEC strains induce the host cell apical cytoskeleton disassembly and the formation of elongated microvilli that surround the attached E. coli, protecting them from phagocytes. Similarly, EPEC induces actin cytoskeleton rearrangements and formation of pedestals reaching up to the adhering E. coli above the surface of enterocytes, and thus protecting them from immune cells. The main targets in the intestinal epithelial barrier for pathogenic groups of E. coli are summarized in Table 1.

In any case, drastic cytoskeleton changes affect the epithelial barrier and have an influence on the redistribution of TJs proteins.

\section{References}

1. Groschwitz KR, Hogan SP. Intestinal barrier function: Molecular regulation and disease pathogenesis. J Allergy Clin Immunol. 2009:124:3-22.

2. Liévin Le-Moal V, Servin AL. Pathogenesis of human enterovirulent bacteria: Lessons from cultured, fully differentiated human colon cancer cell lines. Microbiol Mol Biol Rev. 2013;77:380-439.

3. Peterson LW, Artis D. Intestinal epithelial cells: Regulators of barrier function and immune homeostasis. Nat Rev Immunol. 2014;14:141-153.

4. Blum S, Schiffrin EJ. Intestinal microflora and homeostasis of the mucosal immune response: Implications for probiotic bacteria? Curr Issues Intest Microbiol. 2003;4:53-60.

5. Bischoff S, Barbara G, Buurman W, et al. Intestinal permeability A new target for disease prevention and therapy. BMC Gastroenterol. 2014;14:189-214.

6. van Itallie CM, Holmes J, Bridges $A$, et al. The density of small tight junction pores varies among cell types and is increased by expression of claudin-2. J Cell Sci. 2008;121:298-305.

7. Krause G, Winkler L, Mueller SL, Haseloff RF, Piontek J, Blasig IE. Structure and function of claudins. Biochim Biophys Acta. 2008;1778:631-645.
8. Chiba H, Osanai M, Murata M, Kojima T, Sawada N. Transmembrane proteins of tight junctions. Biochim Biophys Acta. 2008;1778:588-600.

9. Günzel D, Yu AS. Claudins and the modulation of tight junction permeability. Physiol Rev. 2013;93:525-569.

10. Förster $\mathrm{C}$. Tight junctions and the modulation of barrier function in disease. Histochem Cell Biol. 2008;130:55-70.

11. Ebnet K, Suzuki A, Ohno S, Vestweber D. Junctional adhesion molecules (JAMs): More molecules with dual functions? J Cell Sci. 2004;117:19-29.

12. Garrido-Urbani S, Bradfield PF, Imhoff BA. Tight junction dynamics: The role of junctional adhesion molecules (JAMs). Cell Tissue Res. 2014;355(3):701-715. doi: 10.1007/s00441-014-1820-1

13. Gumbiner BM. Regulation of cadherin-mediated adhesion in morphogenesis. Nat Rev Mol Cell Biol. 2005;6:622-634.

14. Perez-Moreno M, Jamora C, Fuchs E. Sticky business: Orchestrating cellular signals at adherens junctions. Cell. 2003;112(4):535-548.

15. Meng W, Takeichi M. Adherens junction: Molecular architecture and regulation. Cold Spring Harb Perspect Biol. 2009;1(6):a002899.

16. Garrod D, Chidgey M. Desmosome structure, composition and function. Biochim Biophys Acta. 2008;1778:572-587.

17. Schmidt A, Koch PJ. Desmosomes. Just cell adhesion or is there more? Cell Adh Migr. 2007;1:28-32.

18. Gassler NC, Rohr A, Schneider J, et al. Inflammatory bowel disease is associated with changes of enterocytic junctions. Am J Physiol Gastrointest Liver Physiol. 2001;281:216-228.

19. Navarro-Garcia F, Serapia-Palacios A, Ugalde-Silva P, Tapia-Pastrana G, Chavez-Duenas L. Actin cytoskeleton manipulation by effector proteins secreted by diarrheagenic Escherichia coli pathotypes. Biomed Res Int. 2013;2013:1-22. doi: 10.1155/2013/374395

20. Brown MD, Bry L, Li Z, Sacks DB. Action pedestal formation by enteropathogenic Escherichia coli is regulated by IQGAP1, calcium, and calmodulin. J Biol Chem. 2008;283:35212-35222.

21. Kim H, White CD, Sacks DB. IQGAP1 in microbial pathogenesis: Targeting the actin cytoskeleton. FEBS Lett. 2011;585:723-729.

22. Peralta-Ramírez J, Hernandez JM, Manning-Cela R, et al. EspF interacts with nucelation-promoting factors to recruit junctional proteins into pedestals for pedestal maturation and disruption of paracellular permeability. Infect Immun. 2008;76:3854-3868.

23. Guttman JA, Kazemi $P$, Lin AE, Vogl AW, Finlay BB. Desmosomes are unaltered during infections by attaching and effacing pathogens. Anat Rec (Hoboken). 2007;290:199-205.

24. Muza-Moons MM, Schneeberger EE, Hecht GA. Enteropathogenic Escherichia coli infection leads to appearance of aberrant tight junctions strands in the lateral membrane of intestinal epithelial cells. Cell Microbiol. 2004;6:783-793.

25. Alto NM, Weflen AW, Rardin MJ, et al. The type III effector EspF coordinates membrane trafficking by the spatiotemporal activation of two eukaryotic signaling pathways. J Cell Biol. 2007;178:1265-1278.

26. Malladi V, Shankar B, Williams PH, Balakrishnan A. Enteropathogenic Escherichia coli outer membrane proteins induce changes in cadherin junctions of Caco-2 cells through activation of PKC alpha. Microbes Infect. 2004;6:38-50.

27. Croxen MA, Finlay BB. Molecular mechanism of Escherichia coli pathogenicity. Nat Rev Microbiol. 2010;8:26-38.

28. Strauman MC, Harper JM, Harrington SM, Boll EJ, Nataro JP. Enteroaggregative Escherichia coli disrupts epithelial tight junctions. Infect Immun. 2010;78:4958-4964.

29. Navarro-García F, Sears C, Eslava C, Cravioto A, Nataro JP. Cytoskeletal effects induced by pet, the serine protease enterotoxin of enteroaggregative Escherichia coli. Infect Immun. 1999;67:2184-2192.

30. Guignot J, Chaplais C, Coconnier-Polter MH, Servin AL. The secreted autotransporter toxin, Sat, functions as a virulence factor in Afa/Dr diffusely adhering Escherichia coli by promoting lesions in tight junction of polarized epithelial cells. Cell Microbiol. 2007;9:204-221.

31. Peiffer I, Blanc-Potard AB, Bernet-Camard MF, Guignot J, Barbat A, Servin AL. Afa/Dr diffusely adhering Escherichia coli C1845 infection promotes selective injuries in the junctional domain of polarized human intestinal Caco-2/TC7 cells. Infect Immun. 2000;68:3431-3442.

32. Kaper JB, Nataro JP, Mobley HL. Pathogenic Escherichia coli. Nat Rev Microbiol. 2004;2:123-140. 
33. Nassour H, Dubreuil JD. Escherichia coli STb enterotoxin dislodges claudin-1 from epithelial tight junctions. Plos One. 2014;9(11):e113273. doi: 10.1371/journal.pone.0113273

34. Mukiza CN, Dubreuil JD. Escherichia coli heat stable toxin b impairs intestinal epithelial barrier function by altering tight junction proteins. Infect Immun. 2013;81:2819-2827.

35. Martinez-Medina M, Garcia-Gil LJ. Escherichia coli in chronic inflammatory bowel disease: An update on adherent invasive Escherichia coli pathogenicity. World J Gastroinest Pathophysiol. 2014;15:213-227.

36. Denizot J, Sivignon A, Barreau F, et al. Adherent-invasive Escherichic coli induce claudin-2 expression and barrier defect in CEABAC10 mice and Crohn's disease patients. Inflamm Bowel Dis. 2012;18:294-304.

37. Sasaki M, Sitaraman SV, Babbin BA, et al. Invasive Escherichia coli are a feature of Crohn's disease. Lab Invest. 2007;87:1042-1054.

38. Wine E, Ossa JC, Gray-Owen SD, Sherman PM. Adherent-invasive Escherichia coli, strain LF82 disrupts apical junctional complexes in polarized epithelia. BMC Microbiol. 2009;9:180-191.

39. Agus A, Massier S, Darfeuille-Michaud A, Billard E, Barnich N. Understanding host adherent-invasive Escherichia coli interaction in Crohn's disease: Opening up new therapeutic strategies. Biomed Research Int. 2014;2014:567929. doi: 10.1155/2014/567929

40. Liévin-Le Moal V, Servin AL. Pathogenesis of human enterovirulent bacteria: Lessons from cultured, fully differentiated human colon cancer cell lines. Microbiol Mol Biol Rev. 2013;77:380-439. 